\title{
Multilevel Correlation Analysis of Influencing Factors on Grain Total Factor Productivity in Main Grain Producing Provinces of China
}

\author{
Bingjun Li, Weiming Yang*, Xiaoxiao Zhu \\ College of Information and Management Science, Henan Agricultural University, Zhengzhou, China \\ Email: *yangweiming626@163.com
}

How to cite this paper: $\mathrm{Li}, \mathrm{B} . J .$, Yang, W.M. and Zhu, X.X. (2018) Multilevel Correlation Analysis of Influencing Factors on Grain Total Factor Productivity in Main Grain Producing Provinces of China. Open Journal of Applied Sciences, 8, 12-24. https://doi.org/10.4236/ojapps.2018.81002

Received: December 14, 2017

Accepted: January 13, 2018

Published: January 16, 2018

Copyright ( 92018 by authors and Scientific Research Publishing Inc. This work is licensed under the Creative Commons Attribution International License (CC BY 4.0).

http://creativecommons.org/licenses/by/4.0/

\begin{abstract}
In order to analyze the influencing factors of TFP (the abbreviation of Total Factor Productivity) deeply, this paper calculates and decomposes the TFP of the main grain producing provinces in China from 2006 to 2015 by the DEA-Malmquist index model. On the basis of this, the grey correlation analysis model based on super-efficiency DEA is used to quantitatively analyze the influencing factors of total factor productivity, technological progress and technical efficiency. The results show that the proportion of grain sowing, the level of food economic development, the level of grain mechanization, the average scale of operations and the level of fertilizer using have the most influence on grain total factor productivity. Finally, according to the results of the analysis, some suggestions are put forward to improve the TFP of different provinces in the main grain producing areas.
\end{abstract}

\section{Keywords}

Malmquist Index, Total Factor Productivity, Super-Efficiency DEA, Grey Correlation Analysis

\section{Introduction}

The grain issue is always the foundation for the development of the country's economy and social stability and an important issue concerning international livelihood for a populous country that accounts for about $20 \%$ of the world's population with $7 \%$ of the world's land. It's an important issue that how to achieve 
sustained growth of grain yield in the process of full urbanization and industrialization with the continued rising prices of means of production, the more and more serious supply of water resources and other resources and the insufficient promotion of agricultural science and technology innovation. TFP is considered as an important comprehensive measure of the growth of food production sexual indicators and the growth of TFP is an important means to promote the steady growth of grain output and achieve the transformation of food production mode. The main grain producing provinces provide about $75 \%$ of the grain for our country every year, which is a powerful backing of grain security and the main source of grain yield in China. Therefore, it is necessary to conduct a quantitative study on grain total factor productivity in main grain producing areas of our country. Grasping its sources of growth and its characteristics and understanding the impact of various factors on total factor productivity of grain have some practical significance to promote the improvement of grain yield and formulate relevant policies for the government.

Many scholars have conducted a multi-faceted study in the field of total factor productivity of grain recent years. Fan calculated the provincial grain total factor productivity from 1978 to 2012 and studied the dynamic evolution of regional disparity in China by the SBM-Malmquist productivity index [1]; Li analyzed the temporal and spatial evolution of the grain total factor productivity and the driving forces in the Yangtze River economic belt from 2001 to 2015 by DEA-malmquist index and Tobit model [2]; Jiao calculated the Malmquist index of grain total productivity by DEA method and analyzed the effect of grain direct subsidy on grain production efficiency based on the provincial panel data of main grain producing areas during 2003-2011 [3]; Ma calculated and decomposed the growth of grain total factor productivity in main producing areas, main selling areas and balanced areas based on the panel data of grain production in 30 provinces of our country from 2001 to 2010 by the method of DEA-Malmquist productivity index [4]. Most of the above studies based on total factor of grain productivity analyze the influencing factors of grain productivity through regression analysis method or macroscopic qualitative method by the panel data of grain production. There are few papers that analyzed the influencing factors of total factor productivity and its decomposition index in the main grain-producing areas by multi-level correlation analysis. First of all, this paper measures and decomposes the TFP of main grain-producing provinces in China by the DEA-Malmquist index model. Then the influencing factors of TFP are quantitative ranking analyzed by the improved grey correlation analysis model based on the super efficiency DEA. The characteristics of many uncertainties in grain production can be fully utilized and the impact of various influencing factors on the grain production can be observed more objectively through the combination of grey correlation analysis and super-efficiency DEA. The local governments of main grain-producing provinces can take more specific measures to improve the efficiency of total factor production of grain by the analysis of this paper. 


\section{Research Methods, Variables and Data}

\subsection{Research Methods}

\subsubsection{DEA-Malmquist Index Model}

The Malmquist index just studied in the theoretical phase and was not initially used in total factor productivity analysis when it was proposed by Sten Malmquist firstly. Then the Malmquist productivity index gradually became a tool to study dynamic efficiency changes while Caves [5] and Charnes [6] constructed the Malmquist productivity index by the distance function ratio. Malmquist productivity index can be decomposed into technical efficiency index and technical progress index in production analysis. The technical efficiency index (EC) can be further decomposed into pure technical efficiency and scale efficiency under the assumption of variable returns to scale.

Malmquist is defined as follows:

$$
M_{i}^{t, t+1}=E C_{i}^{t, t+1} T C_{i}^{t, t+1}, i=1,2, \cdots, n ; t=1,2, \cdots, n
$$

where,

$$
\begin{gathered}
E C_{i}^{t, t+1}=\frac{D_{i}^{t+1}\left(x_{i}^{t+1}+y_{i}^{t+1}\right)}{D_{i}^{t}\left(x_{i}^{t}+y_{i}^{t}\right)} \\
T C_{i}^{t, t+1}=\sqrt{\frac{D_{i}^{t}\left(x_{i}^{t+1}, y_{i}^{t+1}\right)}{D_{i}^{t+1}\left(x_{i}^{t+1}+y_{i}^{t+1}\right)} \times \frac{D_{i}^{t}\left(x_{i}^{t}+y_{i}^{t}\right)}{D_{i}^{t+1}\left(x_{i}^{t}+y_{i}^{t}\right)}}
\end{gathered}
$$

Among them, $x_{i}^{t}$ and $y_{i}^{t}$ represent the input and output of the region of $i$ in the period of $t . D_{i}^{t}\left(x_{i}^{t}+y_{i}^{t}\right)$ and $D_{i}^{t}\left(x_{i}^{t+1}+y_{i}^{t+1}\right)$ are the distance function relative to the technical reference in period of $t$. The Malmquist index of production indicates the degree of change in the productivity of a decision-making unit from one period to another. It indicated that the total factor productivity has been raised during this period when the $M_{i}^{t, t+1} \geq 1$. On the contrary, it indicated that the total factor productivity has been declined. $E C_{i}^{t, t+1}$ indicates the change of technical efficiency of decision-making unit from one period to another and describe the degree of improvement for decision-making unit which relatives to the optimal production front during this period. $E C_{i}^{t, t+1}$ also describes the contribution of the change of technical efficiency to total factor productivity in this period. $T C_{i}^{t, t+1}$ represents the movement of technical frontiers of the decision-making unit. In other words, it represents the contribution of the production frontier movement to the total factor productivity.

\subsubsection{Improved Grey Correlation Model Based on Super Efficiency DEA}

\section{1) Grey Correlation Analysis}

Grey correlation analysis is regarded as an important branch of the grey system theory, the basic idea of the geometric shape of the data sequence is used to determine whether the contact is close, the closer the geometric shape of the sequence curve is, the greater the degree of grey correlation between them is.

Grey system theory is a new method proposed by professor Deng to study the 
problem of the less data and uncertain system. It is based on the evolution of the things with little sample, incomplete information. It can correctly describe and effectively monitor the behavior and evolution of the system through the "part" of the known information generation, development. Grey correlation analysis refers to the quantitative description and comparison of a system development and change situation. The basic idea is to determine whether the relationship between curves is close by determining the similarity between the reference series and the geometric shapes of several comparative series degree [7]. Since the grey correlation analysis model was put forward, many scholars have studied and constructed many grey correlation analysis models [8] [9] [10] [11]. The calculation step of grey correlation analysis can be introduced as follows:

Step 1: Determine the analysis sequence.

Set as the reference sequence:

$$
X_{0}=\left(x_{0}(1), x_{0}(2), \cdots, x_{0}(n)\right)
$$

Set as the comparison sequence:

$$
X_{i}=\left(x_{i}(1), x_{i}(2), \cdots, x_{i}(n)\right)(i=1,2, \cdots, m)
$$

Step 2: Nondimensionalize the original data.

The initialized reference sequence is:

$$
X_{0}^{\prime}=x_{0}(k) / x_{0}(1)=\left(x_{0}(1), x_{0}(2), \cdots, x_{0}(n)\right)(i=1,2, \cdots, m)
$$

The initialized comparison sequence is:

$$
X_{i}^{\prime}=X_{i}(k) / x_{i}(1)=\left(x_{i}^{\prime}(1), x_{i}^{\prime}(2), \cdots, x_{i}^{\prime}(n)\right)(i=1,2, \cdots, m)
$$

Step 3: Calculate difference sequence.

The calculation formula of sequence difference is:

$$
\Delta_{0 i}(k)=\left|x_{0}(k)-x_{i}(k)\right|(i=1,2,3, \cdots, m, k=1,2,3, \cdots, n)
$$

The maximum and minimum calculated by Formula (5) are:

Maximum: $M=\max _{i} \max _{k} \Delta_{i}(k)=\Delta_{\max }$.

Minimum: $m=\min _{i} \min _{k} \Delta_{i}(k)=\Delta_{\min }$.

Step 4: Calculate grey correlation coefficient.

The calculation formula of grey correlation coefficient is:

$$
\gamma_{0 i}(k)=\frac{m+\rho M}{\Delta_{0 i}(k)+\rho M}
$$

where, $\rho \in(0,1), i=1,2,3, \cdots, m, \rho$ is called resolution coefficient, set $\rho=0.5$ usually.

Step 5: Calculate grey correlation degree.

The calculation formula of grey correlation degree is:

$$
\gamma_{0 i}=\frac{1}{n} \sum_{k=1}^{n} \gamma_{0 i}(k)
$$

The $\gamma_{0 i}$ is the comparison sequence $x_{i}$ to reference sequence $x_{0}$, which 
can reveal the degree of their association.

\section{2) Super Efficiency DEA}

The DEA model is a non-parametric measurement method that measures the level of efficiency of a production unit by calculating the distance between the actual output of the production unit and the production frontiers. However, the traditional DEA model cannot be further evaluated when there are multiple effective decision making units. Therefore, Andersen [12] improved the traditional DEA model and proposed the super-efficient DEA model, which solved the sorting problem of effective unit. The super efficient DEA model can be divided into input-oriented and output-oriented according to different perspectives. This paper adopts an input-oriented super-efficient DEA model. The expression is as follows:

$$
\left\{\begin{array}{l}
\max h_{0}=\sum_{r=1}^{s} \mu_{r} y_{r 0} \\
\text { s.t. } \sum_{i=1}^{m} \omega_{i} x_{0}=1 \\
\sum_{i=1}^{m} \omega_{i} x_{i j}-\sum_{r=1}^{s} \mu_{r} y_{r j} \geq 0 \\
\omega_{i, \mu r} \geq 0, \forall i, r(j=1,2,3, \cdots, n)
\end{array}\right.
$$

\section{3) Grey Correlation Model Based on Super Efficiency DEA}

$\mathrm{Li}$ and Liu [13] [14] improves the grey correlation analysis by the above super-efficiency DEA model to calculate the $r_{0, i}$. In this model, you can construct the $\mathrm{n}$-dimensional input vector whose input value is 1 , regard each attribute as a decision unit, and the corresponding output value is the relevance value of this point. The grey correlation analysis can be improved to obtain the following mixed model by being combined with super-efficiency DEA:

$$
\left\{\begin{array}{l}
\max r_{0, i}=\sum_{t=1}^{l} \omega_{t} \times \xi_{0,1}(t) \\
\text { s.t. } \sum_{t=1}^{l} \omega_{t} \xi_{0, i} \leq 1 \\
i=1,2, \cdots, n\left(i \neq i_{0}\right) \\
\omega_{t} \geq 0, \forall t
\end{array}\right.
$$

We can get the weight and the optimal grey correlation of the $i_{0}$ by solving the formula above. The corresponding weight vector of each factor has been given in (9), which makes the analysis results more objective compared with the traditional grey correlation analysis.

\subsection{Data Source}

The data in this paper are the panel data which related to the input, output and influencing factors of grain production of main grain producing areas in China from 2006 to 2015. Some data in the paper come from "China Statistical Yearbook", "China Rural Statistical Yearbook" and "Compilation of Rural Data in 
the Past Thirty Years from Reform and Opening Up", some data are calculated based on the data of statistical yearbook.

\subsection{Variable Description}

These indexes in the paper are chosen by referencing to the existing research literature and the indexes are measurable or accessible [15] [16] [17] [18] [19]. The output index in the paper is grain yield of the main grain-producing provinces. This article divests the grain production inputs from generalized agriculture by the weight coefficient method due to the lack of statistical data on the separate grain production factors. The five input indicators are labor input (million people), calculated by the number of employees engaged in agriculture, forestry, animal husbandry and fishery in the provinces, excluding the labor force engaged in industries and service industries in rural areas; land input (thousand hectares), calculated on the total power of agricultural machinery; input of chemical fertilizers (10,000 tons), calculated based on the amount of chemical fertilizer actually used for agricultural production during this year (one thousand hectares); pesticide inputs (thousand hectares),calculated by the use (million tons) of annual pesticide.

\section{Analysis of Total Factor Productivity in Main Grain Producing Provinces}

The Malmquist productivity indexs in 13 provinces of the main grain-producing areas from 2006 to 2015 are shown in Table 1 by the DEAP2.1.

Table 1. Changes and decompositions of total factor productivity of different units in main grain producing areas in China from 2006 to 2015.

\begin{tabular}{cccccc}
\hline & $\begin{array}{c}\text { Efficiency } \\
\text { change }\end{array}$ & $\begin{array}{c}\text { Technological } \\
\text { change }\end{array}$ & $\begin{array}{c}\text { Pure } \\
\text { technological } \\
\text { efficiency change }\end{array}$ & $\begin{array}{c}\text { Scale } \\
\text { efficiency } \\
\text { change }\end{array}$ & $\begin{array}{c}\text { Total factor } \\
\text { efficiency } \\
\text { change }\end{array}$ \\
\hline Anhui & 1.010 & 1.008 & 1.007 & 1.003 & 1.018 \\
Hebei & 1.007 & 1.011 & 1.006 & 1.001 & 1.018 \\
Heilongjiang & 1.000 & 1.016 & 1.000 & 1.000 & 1.016 \\
Hubei & 1.000 & 1.029 & 1.000 & 1.000 & 1.029 \\
Hunan & 0.994 & 1.010 & 0.995 & 0.999 & 1.003 \\
Jilin & 1.000 & 0.991 & 1.000 & 1.000 & 0.991 \\
Jiangsu & 1.000 & 1.010 & 1.000 & 1.000 & 1.010 \\
Jiangxi & 1.002 & 1.006 & 1.025 & 0.978 & 1.009 \\
Liaoning & 0.994 & 1.008 & 1.000 & 0.994 & 1.001 \\
Inner Mongolia & 1.000 & 0.986 & 1.000 & 1.000 & 0.986 \\
Shandong & 0.998 & 1.011 & 1.000 & 0.998 & 1.008 \\
Sichuan & 1.016 & 1.005 & 1.000 & 1.016 & 1.021 \\
Henan & 0.998 & 1.014 & 1.000 & 0.998 & 1.011 \\
Mean & 1.001 & 1.008 & 1.002 & 0.999 & 1.009 \\
\hline
\end{tabular}

Note: The data in Table 1 come from the calculate of the data from "China Statistical Yearbook", "China Rural Statistical Yearbook" and "Compilation of Rural Data in the Past Thirty Years from Reform and Opening Up". 
From Table 1, we can find that eleven provinces' grain total factor productivity has risen from 2006 to 2015 with the exception of Jilin Province and Inner Mongolia, where the annual average decreases $0.9 \%$ and $1.4 \%$. Among which, the total factor productivity of Hubei Province rose fastest, followed by Anhui Province and Hebei Province.

In terms of the decomposition of total factor productivity, the technological progress in Hubei Province was 2.9\% from 2006 to 2015, which was the fastest in main grain producing provinces and Heilongjiang Province was $1.6 \%$ followed. Only technological progress of Jilin Province and Inner Mongolia decreased by $0.9 \%$ and $1.4 \%$ respectively. The technical efficiency of Anhui Province, Hebei Province, Jiangxi Province and Sichuan Province increased by an average of $1 \%$, $0.7 \%, 0.2 \%$ and $1.6 \%$ respectively; Technical efficiency of Hunan Province, Liaoning Province, Shandong Province and Henan Province decreased by $0.6 \%$, $0.6 \%, 0.2 \%$ and $0.2 \%$ respectively; The technical efficiency of Heilongjiang Province, Hubei Province, Jilin Province, Jiangsu Province and Inner Mongolia remained unchanged. The technical efficiency can be further decomposed into pure technical efficiency and scale efficiency. It can be seen that the purely technological efficiency increases in Anhui Province, Hebei Province and Jiangxi Province by $0.7 \%, 0.6 \%$ and $2.5 \%$ respectively; while purely technological efficiency declined by $0.5 \%$ in Hunan Province and the remaining province have not changed. The scale efficiency increased by $0.3 \%, 0.1 \%$ and $1.6 \%$ in Anhui Province, Hebei Province, Sichuan Province and decreased by $0.1 \%, 2.2 \%, 0.6 \%$ and $0.2 \%$ in Hunan Province, Jiangxi Province, Liaoning Province, Shandong Province and Henan Province, the remaining provinces have not changed.

\section{Multilevel Correlation Analysis of Influencing Factors on Total Factor Productivity}

\subsection{Selection of the Index of Influencing Factors of Grain Total Factor Productivity}

1) Grain yield per unit area $\left(X_{1}\right)$ : It's expressed as the output of grain in a certain area divided by the total sown area of grain. The increasing of grain yield per unit area means the grain production efficiency increasing. Therefore, there are relevance among the per unit area of grain production and total factor productivity of grain, food production efficiency and technological change.

2) Proportion of grain sown $\left(X_{2}\right)$ : It is expressed by the proportion of sown area of grain in the total sown area of the crop. It reflects the level of enthusiasm for sowing grain in the area.

3) Average labor management scale $\left(X_{3}\right)$ : It is expressed that the sown area of grain in a certain area divided by the number of grain laborers (the number of grain laborers $=$ the number of agricultural workers ${ }^{*}$ the sown area of grain/the sown area of crops).

4) The level of fertilizer using per unit area $\left(X_{4}\right)$ : It is expressed by the amount of pure fertilizers divided by the sown area of the crop in a region. 
Within a reasonable range, the higher the proportion is, the higher the growth of grain crops will be.

5) The development level of grain economy $\left(X_{5}\right)$ : It is expressed as the ratio of the gross value of agricultural output to the total output value of agriculture, forestry, husbandry and fishery in a certain region and then multiplied by the ratio of sown area of food crops to sown area of crops. The higher the ratio is, the higher the level of grain economy development will be. In general, the higher the level of grain economy development, the more developed the grain industry and the higher the total factor productivity of food will be.

6) The level of afflicted crops $\left(X_{6}\right)$ : It is measured by the ratio of the disaster area of crops to total sown area of crops in an area. In order to accurately measure the level of disaster of food crops, the affected area of crops multiplied by the ratio of the sown area of grain crop to the sown area of crop. The higher the ratio is, the more serious the disaster is and the less promotion to the improvement of grain production efficiency.

7) The level of grain mechanization $\left(X_{7}\right)$ : It is expressed by the ratio of the total agricultural machinery to the total crop sown area. This index reflects the substitution ability of labor resources. The higher the ratio is, the more obvious the substitution of labor resources will be.

According to the selection of Index and grey correlation method based on super efficiency DEA, we can calculate the correlation degree and the ranking of factors affecting the Total Factor Productivity of Grain, Technological Progress and Technical Efficiency. The results are shown in Table 2 and Table 3.

\subsection{Analysis of Influence Factors}

According to the ranking of influencing factors on total factor productivity of the main grain producing areas, Anhui Province, Hubei Province, Inner Mongolia, Sichuan Province and Henan Province were most affected by the sown proportion of grain; Hebei Province, Heilongjiang Province, Hunan Province and Jiangsu Province, Jilin Province and Liaoning Province were most affected by the level of grain economy development; Jilin Province and Liaoning Province were most affected by the level of grain mechanization; Jiangxi Province was most affected by the average labor management scale; Shandong Province was most affected by the level of fertilizer using per unit area.

According to the ranking of the influencing factors on technological progress province of the main grain producing areas, Anhui Province, Jiangxi Province and Sichuan Province were most affected by the average labor management scale; Heilongjiang Province, Hunan Province, Jiangsu Province and Henan Province were most affected by the level of grain economy development; Hubei Province, Liaoning Province, Inner Mongolia and Shandong Province were most affected by the proportion of grain sown; Hebei Province was most affected by the level of fertilizer using per unit area; Jilin Province was most affected by the level of grain mechanization. 
Table 2. Correlation degree of factors affecting the total factor productivity of grain, technological progress and technical efficiency.

\begin{tabular}{|c|c|c|c|c|c|c|c|c|}
\hline Province & & $\gamma_{1}$ & $\gamma_{2}$ & $\gamma_{3}$ & $\gamma_{4}$ & $\gamma_{5}$ & $\gamma_{6}$ & $\gamma_{7}$ \\
\hline \multirow{3}{*}{ Anhui } & tfpch & 0.705 & 0.920 & 0.904 & 0.906 & 0.919 & 0.794 & 0.834 \\
\hline & techch & 0.659 & 0.964 & 0.982 & 0.910 & 0.964 & 0.729 & 0.760 \\
\hline & effch & 0.711 & 0.908 & 0.893 & 0.908 & 0.908 & 0.802 & 0.843 \\
\hline \multirow{3}{*}{ Hebei } & tfpch & 0.641 & 0.970 & 0.886 & 0.959 & 0.985 & 0.867 & 0.863 \\
\hline & techch & 0.655 & 0.928 & 0.923 & 0.959 & 0.955 & 0.896 & 0.898 \\
\hline & effch & 0.668 & 0.896 & 0.931 & 0.929 & 0.920 & 0.892 & 0.931 \\
\hline \multirow{3}{*}{ Heilongjiang } & tfpch & 0.622 & 0.819 & 0.903 & 0.844 & 0.929 & 0.813 & 0.863 \\
\hline & techch & 0.622 & 0.819 & 0.903 & 0.844 & 0.929 & 0.813 & 0.863 \\
\hline & effch & 0.575 & 0.975 & 0.893 & 0.863 & 0.798 & 0.775 & 0.719 \\
\hline \multirow{3}{*}{ Hubei } & tfpch & 0.712 & 0.905 & 0.828 & 0.835 & 0.857 & 0.850 & 0.895 \\
\hline & techch & 0.712 & 0.905 & 0.828 & 0.835 & 0.857 & 0.850 & 0.895 \\
\hline & effch & 0.689 & 0.899 & 0.990 & 0.980 & 0.952 & 0.725 & 0.754 \\
\hline \multirow{3}{*}{ Hunan } & tfpch & 0.729 & 0.950 & 0.959 & 0.897 & 0.960 & 0.716 & 0.874 \\
\hline & techch & 0.729 & 0.950 & 0.959 & 0.897 & 0.960 & 0.716 & 0.874 \\
\hline & effch & 0.731 & 0.970 & 0.943 & 0.969 & 0.962 & 0.682 & 0.816 \\
\hline \multirow{3}{*}{ Jilin } & tfpch & 0.709 & 0.815 & 0.860 & 0.852 & 0.810 & 0.814 & 0.888 \\
\hline & techch & 0.709 & 0.815 & 0.860 & 0.852 & 0.810 & 0.814 & 0.888 \\
\hline & effch & 0.613 & 0.920 & 0.881 & 0.859 & 0.929 & 0.742 & 0.728 \\
\hline \multirow{3}{*}{ Jiangsu } & tfpch & 0.714 & 0.962 & 0.896 & 0.955 & 0.976 & 0.927 & 0.832 \\
\hline & techch & 0.715 & 0.962 & 0.896 & 0.954 & 0.976 & 0.927 & 0.833 \\
\hline & effch & 0.690 & 0.979 & 0.850 & 0.987 & 0.950 & 0.918 & 0.794 \\
\hline \multirow{3}{*}{ Jiangxi } & tfpch & 0.746 & 0.825 & 0.892 & 0.841 & 0.819 & 0.683 & 0.825 \\
\hline & techch & 0.744 & 0.814 & 0.900 & 0.831 & 0.809 & 0.691 & 0.836 \\
\hline & effch & 0.671 & 0.733 & 0.885 & 0.744 & 0.729 & 0.753 & 0.953 \\
\hline \multirow{3}{*}{ Liaoning } & tfpch & 0.664 & 0.840 & 0.916 & 0.858 & 0.865 & 0.871 & 0.933 \\
\hline & techch & 0.617 & 0.983 & 0.931 & 0.936 & 0.956 & 0.873 & 0.813 \\
\hline & effch & 0.652 & 0.869 & 0.938 & 0.887 & 0.895 & 0.875 & 0.911 \\
\hline \multirow{3}{*}{ Inner Mongolia } & tfpch & 0.603 & 0.984 & 0.906 & 0.877 & 0.954 & 0.948 & 0.867 \\
\hline & techch & 0.603 & 0.984 & 0.906 & 0.877 & 0.954 & 0.948 & 0.867 \\
\hline & effch & 0.591 & 0.954 & 0.885 & 0.879 & 0.984 & 0.903 & 0.823 \\
\hline \multirow{3}{*}{ Shandong } & tfpch & 0.729 & 0.963 & 0.875 & 0.964 & 0.899 & 0.696 & 0.805 \\
\hline & techch & 0.744 & 0.988 & 0.900 & 0.964 & 0.926 & 0.709 & 0.825 \\
\hline & effch & 0.725 & 0.956 & 0.868 & 0.964 & 0.893 & 0.693 & 0.800 \\
\hline \multirow{3}{*}{ Sichuan } & tfpch & 0.706 & 0.994 & 0.839 & 0.985 & 0.982 & 0.672 & 0.755 \\
\hline & techch & 0.695 & 0.817 & 0.926 & 0.805 & 0.803 & 0.777 & 0.880 \\
\hline & effch & 0.678 & 0.802 & 0.912 & 0.790 & 0.788 & 0.786 & 0.891 \\
\hline \multirow{3}{*}{ Henan } & tfpch & 0.743 & 0.983 & 0.813 & 0.916 & 0.971 & 0.869 & 0.867 \\
\hline & techch & 0.748 & 0.974 & 0.801 & 0.916 & 0.977 & 0.873 & 0.853 \\
\hline & effch & 0.723 & 0.974 & 0.769 & 0.923 & 0.958 & 0.835 & 0.816 \\
\hline
\end{tabular}


Table 3. Ranks of factors affecting the total factor productivity of grain, technological progress and technical efficiency.

\begin{tabular}{|c|c|c|}
\hline Province & & Correlation degree ranking of influential factors \\
\hline \multirow{3}{*}{ Anhui } & tfpch & $\gamma_{2} \succ \gamma_{5} \succ \gamma_{4} \succ \gamma_{3} \succ \gamma_{7} \succ \gamma_{6} \succ \gamma_{1}$ \\
\hline & techch & $\gamma_{3} \succ \gamma_{2} \succ \gamma_{5} \succ \gamma_{4} \succ \gamma_{7} \succ \gamma_{6} \succ \gamma_{1}$ \\
\hline & effch & $\gamma_{4} \succ \gamma_{2} \succ \gamma_{5} \succ \gamma_{3} \succ \gamma_{7} \succ \gamma_{6} \succ \gamma_{1}$ \\
\hline \multirow{3}{*}{ Hebei } & tfpch & $\gamma_{5} \succ \gamma_{2} \succ \gamma_{4} \succ \gamma_{3} \succ \gamma_{6} \succ \gamma_{7} \succ \gamma_{1}$ \\
\hline & techch & $\gamma_{4} \succ \gamma_{5} \succ \gamma_{2} \succ \gamma_{3} \succ \gamma_{7} \succ \gamma_{6} \succ \gamma_{1}$ \\
\hline & effch & $\gamma_{3} \succ \gamma_{7} \succ \gamma_{4} \succ \gamma_{5} \succ \gamma_{2} \succ \gamma_{6} \succ \gamma_{1}$ \\
\hline \multirow{3}{*}{ Heilongjiang } & tfpch & $\gamma_{2} \succ \gamma_{7} \succ \gamma_{5} \succ \gamma_{6} \succ \gamma_{4} \succ \gamma_{3} \succ \gamma_{1}$ \\
\hline & techch & $\gamma_{5} \succ \gamma_{3} \succ \gamma_{7} \succ \gamma_{4} \succ \gamma_{2} \succ \gamma_{6} \succ \gamma_{1}$ \\
\hline & effch & $\gamma_{2} \succ \gamma_{3} \succ \gamma_{4} \succ \gamma_{5} \succ \gamma_{6} \succ \gamma_{7} \succ \gamma_{1}$ \\
\hline \multirow{3}{*}{ Hubei } & tfpch & $\gamma_{2} \succ \gamma_{7} \succ \gamma_{5} \succ \gamma_{6} \succ \gamma_{4} \succ \gamma_{3} \succ \gamma_{1}$ \\
\hline & techch & $\gamma_{2} \succ \gamma_{7} \succ \gamma_{5} \succ \gamma_{6} \succ \gamma_{4} \succ \gamma_{3} \succ \gamma_{1}$ \\
\hline & effch & $\gamma_{3} \succ \gamma_{4} \succ \gamma_{5} \succ \gamma_{2} \succ \gamma_{7} \succ \gamma_{6} \succ \gamma_{1}$ \\
\hline \multirow{3}{*}{ Hunan } & tfpch & $\gamma_{2} \succ \gamma_{5} \succ \gamma_{4} \succ \gamma_{3} \succ \gamma_{7} \succ \gamma_{6} \succ \gamma_{1}$ \\
\hline & techch & $\gamma_{5} \succ \gamma_{3} \succ \gamma_{2} \succ \gamma_{4} \succ \gamma_{7} \succ \gamma_{1} \succ \gamma_{6}$ \\
\hline & effch & $\gamma_{2} \succ \gamma_{4} \succ \gamma_{5} \succ \gamma_{3} \succ \gamma_{7} \succ \gamma_{1} \succ \gamma_{6}$ \\
\hline \multirow{3}{*}{ Jilin } & tfpch & $\gamma_{7} \succ \gamma_{3} \succ \gamma_{4} \succ \gamma_{2} \succ \gamma_{6} \succ \gamma_{5} \succ \gamma_{1}$ \\
\hline & techch & $\gamma_{7} \succ \gamma_{3} \succ \gamma_{4} \succ \gamma_{2} \succ \gamma_{6} \succ \gamma_{5} \succ \gamma_{1}$ \\
\hline & effch & $\gamma_{5} \succ \gamma_{2} \succ \gamma_{3} \succ \gamma_{4} \succ \gamma_{6} \succ \gamma_{7} \succ \gamma_{1}$ \\
\hline \multirow{3}{*}{ Jiangsu } & tfpch & $\gamma_{5} \succ \gamma_{2} \succ \gamma_{4} \succ \gamma_{6} \succ \gamma_{3} \succ \gamma_{7} \succ \gamma_{1}$ \\
\hline & techch & $\gamma_{5} \succ \gamma_{2} \succ \gamma_{4} \succ \gamma_{6} \succ \gamma_{3} \succ \gamma_{7} \succ \gamma_{1}$ \\
\hline & effch & $\gamma_{4} \succ \gamma_{6} \succ \gamma_{3} \succ \gamma_{2} \succ \gamma_{5} \succ \gamma_{1} \succ \gamma_{7}$ \\
\hline \multirow{3}{*}{ Jiangxi } & tfpch & $\gamma_{3} \succ \gamma_{4} \succ \gamma_{7} \succ \gamma_{2} \succ \gamma_{5} \succ \gamma_{1} \succ \gamma_{6}$ \\
\hline & techch & $\gamma_{3} \succ \gamma_{7} \succ \gamma_{4} \succ \gamma_{2} \succ \gamma_{5} \succ \gamma_{1} \succ \gamma_{6}$ \\
\hline & effch & $\gamma_{7} \succ \gamma_{3} \succ \gamma_{6} \succ \gamma_{4} \succ \gamma_{2} \succ \gamma_{5} \succ \gamma_{1}$ \\
\hline \multirow{3}{*}{ Liaoning } & tfpch & $\gamma_{7} \succ \gamma_{3} \succ \gamma_{6} \succ \gamma_{5} \succ \gamma_{4} \succ \gamma_{2} \succ \gamma_{1}$ \\
\hline & techch & $\gamma_{2} \succ \gamma_{5} \succ \gamma_{4} \succ \gamma_{3} \succ \gamma_{6} \succ \gamma_{7} \succ \gamma_{1}$ \\
\hline & effch & $\gamma_{3} \succ \gamma_{7} \succ \gamma_{5} \succ \gamma_{4} \succ \gamma_{6} \succ \gamma_{2} \succ \gamma_{1}$ \\
\hline \multirow{3}{*}{ Inner Mongolia } & tfpch & $\gamma_{2} \succ \gamma_{5} \succ \gamma_{6} \succ \gamma_{3} \succ \gamma_{4} \succ \gamma_{7} \succ \gamma_{1}$ \\
\hline & techch & $\gamma_{2} \succ \gamma_{5} \succ \gamma_{6} \succ \gamma_{3} \succ \gamma_{4} \succ \gamma_{7} \succ \gamma_{1}$ \\
\hline & effch & $\gamma_{5} \succ \gamma_{2} \succ \gamma_{6} \succ \gamma_{3} \succ \gamma_{4} \succ \gamma_{7} \succ \gamma_{1}$ \\
\hline
\end{tabular}




\section{Continued}

\begin{tabular}{ll}
\hline tfpch & $\gamma_{4} \succ \gamma_{2} \succ \gamma_{5} \succ \gamma_{3} \succ \gamma_{7} \succ \gamma_{1} \succ \gamma_{6}$ \\
\hline Shandong & $\gamma_{2} \succ \gamma_{4} \succ \gamma_{5} \succ \gamma_{3} \succ \gamma_{7} \succ \gamma_{1} \succ \gamma_{6}$ \\
effch & $\gamma_{4} \succ \gamma_{2} \succ \gamma_{5} \succ \gamma_{3} \succ \gamma_{7} \succ \gamma_{1} \succ \gamma_{6}$ \\
tfpch & $\gamma_{2} \succ \gamma_{4} \succ \gamma_{5} \succ \gamma_{3} \succ \gamma_{7} \succ \gamma_{1} \succ \gamma_{6}$ \\
techch & $\gamma_{3} \succ \gamma_{7} \succ \gamma_{2} \succ \gamma_{4} \succ \gamma_{5} \succ \gamma_{6} \succ \gamma_{1}$ \\
effch & $\gamma_{3} \succ \gamma_{7} \succ \gamma_{2} \succ \gamma_{4} \succ \gamma_{5} \succ \gamma_{6} \succ \gamma_{1}$ \\
tfpch & $\gamma_{2} \succ \gamma_{5} \succ \gamma_{4} \succ \gamma_{6} \succ \gamma_{7} \succ \gamma_{3} \succ \gamma_{1}$ \\
techch & $\gamma_{5} \succ \gamma_{1} \succ \gamma_{4} \succ \gamma_{6} \succ \gamma_{7} \succ \gamma_{3} \succ \gamma_{1}$ \\
Henan & $\gamma_{2} \succ \gamma_{5} \succ \gamma_{4} \succ \gamma_{6} \succ \gamma_{7} \succ \gamma_{3} \succ \gamma_{1}$ \\
\hline
\end{tabular}

Note: In Table 2 and Table 3, the data come from the calculate of the data from "China Statistical Yearbook", "China Rural Statistical Yearbook" and "Compilation of Rural Data in the Past Thirty Years from Reform and Opening Up". The "tfpch", "techch" and "effch" are the abbreviation of change of technology, efficiency and TFP. $\gamma_{1}-\gamma_{7}$ represents the correlation degree between $X_{1}-X_{7}$ and tfpch, techch, effch.

In terms of the ranking of the influencing factors on technical efficiency of the main grain producing areas, Hebei Province, Hubei Province, Liaoning Province and Sichuan Province were most affected by the average labor management scale; Heilongjiang Province, Hunan Province and Henan Province were greatest impacted by the proportion of grain sown; Jilin Province, Inner Mongolia were most affected by the level of grain economy development; Anhui Province, Jiangsu Province and Shandong Province were most impacted by the level of fertilizer using per unit area; Jiangxi Province was greatest impacted by the level of grain mechanization.

According to the tendency of the overall ranking of the various factors, the trend of technological progress and total factor productivity are the closest. It shows that the total factor productivity is "dominated by technological progress" on the other hand. All local governments can make corresponding adjustments according to the specific sequencing of the province and improve the grain total factor productivity overall.

\section{Conclusions}

It is possible to make a comprehensive judgment of the factors that affect the TFP of main grain producing provinces from various angles and make various policies according to the local conditions through the multilevel grey correlation analysis of the decomposition of total factor productivity and the influencing factors.

In order to further promote the grain total factor productivity in the main grain producing areas, improvements can be made in the following aspects: The government of Anhui Province, Hubei Province, Inner Mongolia, Sichuan Province and Henan Province should optimize the grain production structure 
and increase grain sown area. It is necessary to make more rational use of land for grain production based on comprehensive consideration of the production conditions of climate, land and resources in various regions, the actual production all over the country. In order to increase the proportion of grain sown, these provinces should make full use of land resources; Hebei Province, Heilongjiang Province, Hunan Province and Jiangsu Province should increase the gross value of agricultural production, increase their investment in scientific research on grain production, establish a sound grain subsidy system, raise the overall level of grain economy and promote the development of grain industry; Jilin Province and Liaoning Province should focus on improving the scale and mechanization of grain production to increase the grain production efficiency; Jiangxi Province should step up its efforts to support grain production and increase various kinds of policies to benefit farmers so as to raise farmers' enthusiasm for grain cultivation and actively create various kinds of jobs and training for peasants; Shandong Province should increase the scientific and technological research of grain and the cultivation of new varieties. The government should guide peasants to use chemical fertilizers in a scientific and rational manner to increase grain yield per unit area.

In addition, in order to achieve the "double-layer" promotion of technological progress and technological efficiency for all-provincial grain total factor productivity, governments at all levels can prioritize technological progress and enhance technological efficiency according to the ranking of factors influencing technological progress and the factors influencing technological efficiency. On the basis of the research in this paper, other scholars can further study the relationship between TFP and technological progress and technical efficiency so as to better solve the problem of TFP' increase.

\section{Funding Information}

The work was supported in part by the Soft-science Foundation of Henan Province (172400410015); Philosophy and Social Science Program of Henan Province (2016BJJ022).

\section{References}

[1] Fan, L.X. (2017) Distribution Dynamics and Trend Evolution of Total Factor Productivity in China-Empirical Evidence Based on Provincial Panel Data from 1978 to 2012. Rural Economy, 3, 49-54.

[2] Li, L.L. and Zhang, L.G. (2017) Empirical Analysis of the Spatial-temporal Evolution and Driving Factor of Grain Total Factor Productivity of Yangtze River Economic Belt. Prices Monthly, 6, 77-82.

[3] Jiao, J.P. and Song, X.H. (2015) Empirical Analysis on the Factors Influencing of Grain Total Factor Productivity. Statistics \& Decision, 11, 126-129.

[4] Ma, L.J., Wang, Y.P. and Tian, Y. (2014) Regional Differentiation of Grain Total Factor Productivity and Influencing Factors in China. Research of Agricultural Modernization, 4, 385-391. 
[5] Caves, D.W., Christensen, L.R. and Diewert, W.E. (1982) The Economic Theory of Index Numbers and the Measurement of Input, Output, and Productivity. Ecomometreca, 50, 1393-1414. https://doi.org/10.2307/1913388

[6] Charnes, A. and Cooper, W.W. (1978) Measuring the Efficiency of Decision Making Units. European Journal of Operational Research, 2, 429-444. https://doi.org/10.1016/0377-2217(78)90138-8

[7] Liu, S.F., Yang, Y.J. and Jeffrey, F. (2017) Grey Data Analysis: Methods, Models and Applications. Springer-Verlag, Singapore. https://doi.org/10.1007/978-981-10-1841-1

[8] Liu, S.F., Forrest, J. and Yang, Y.J. (2013) Advances in Grey Systems Research. Journal of Grey System, 2, 1-18. https://doi.org/10.1108/GS-09-2015-0054

[9] Liu, S.F., Yang, Y.J. and Xie, N.M. (2016) New Progress of Grey System Theory in the New Millennium. Grey Systems Theory and Application, 1, 2-31.

[10] Zhang, K., Zhong, Q.P. and Zuo, Y. (2017) Multivariate Grey Gradient Incidence Model and Its Application. Grey Systems: Theory and Application, 7, 236-246. https://doi.org/10.1108/GS-04-2017-0007

[11] Liu, S.F., Tao, L. and Xie, N.M. (2016) On the New Model System and Framework of Grey System Theory. The Journal of Grey System, 1, 1-15.

[12] Andersen, P. and Petersen, N.C. (1993) A Procedure for Ranking Efficient Units in Data Envelopment Analysis. Management Science, 39, 1261-1264.

https://doi.org/10.1287/mnsc.39.10.1261

[13] Li, L., Liu, J.Y., Fu, C.Q. and Wu, Z.J. (2010) MCDM Model Based on Grey Correlation Analysis and Super-Efficiency DEA. Application Research of Computers, 2, 526-528.

[14] Liu, N. (2011) Evaluation on the Grain Production Capacity of Major Grain Production Area Based on Super-efficiency Output-DEA Model. Soft Science, 3, 79-83.

[15] Fan, L.X. (2016) A Study on Growth and Decomposition of China's Grain TFP Growth. Economic Survey, 3, 31-36.

[16] Yang, J.Y., Han, X.N. and Fang, X.M. (2013) Empirical Study on the Efficiency of Grain Production in China. Economic Perspectives, 6, 47-53.

[17] Xue, L. and Liu, Q. (2013) Analysis of Food Production Technical Efficiency and Total Factor Productivity of Henan Province. Journal of Henan Agricultural University, 3, 345-350.

[18] Zhou, M.H. (2010) An Empirical Study on the Total Factor Product Food Production in China: 1978-2010. Journal of Guangdong University of Business Studies, 2, 70-76.

[19] Wei, D., Min, R. and Wang, Y.P. (2010) Technical Efficiency, Technical Progress and Grain Productivity Growth-an Empirical Analysis Based on Provincial Panel Data in China. Forum on Science and Technology in China, 8, 140-145. 